\title{
Different approach to pulsed high-voltage vacuum-insulation design
}

\author{
John G. Leopold, Chaim Leibovitz, Itamar Navon, and Meir Markovits \\ Department of Applied Physics, Rafael Laboratories, P.O. Box 2250, Haifa 31021, Israel
}

(Received 8 May 2007; published 5 June 2007)

\begin{abstract}
A theoretical methodology promising improved design of vacuum insulation in high-voltage pulsedpower systems is described. It consists of shaping the electromagnetic fields within the system in such a way that charged particles which can in principle initiate vacuum surface breakdown are deflected away from the insulator surface, and secondary electrons, if emitted, are prevented from restriking the surface. Thus, vacuum surface breakdown is prevented before it is able to develop. Our methodology is presented here by a set of case studies.
\end{abstract}

DOI: 10.1103/PhysRevSTAB.10.060401

PACS numbers: 84.70.+p, 41.90.+e, 77.90.+k, 41.20.- $\mathrm{q}$

\section{INTRODUCTION}

Vacuum surface breakdown [1] over the insulator surface between conducting regions separated by a highvoltage gap is the major limiting factor in the design of high-voltage vacuum systems.

The traditional approach to the design of vacuum insulation in pulsed-power systems is to avoid the formation of elevated electric fields on the insulator surface [2-4]. To decrease the probability of vacuum surface breakdown along the surface, it is accepted practice to increase the area of insulators so that voltage gradients are decreased and the longest possible flashover routes are achieved [5]. It is also common practice to screen triple junctions, that is, the interface where vacuum, the insulator, and the conductor surfaces meet [6]. Triple junctions are known to be major breakdown initiating regions [1]. Other methods attempt to decrease secondary electron multiplication by either magnetic steering of secondaries away from the insulator surface [7], or by applying special coatings on the surface which suppress electron multiplication [8].

The mechanism for the development of the flashover is complex and it can involve unpredictable primary processes such as the explosion of microwhiskers on imperfect metal surfaces, the ionization of gas remnants of imperfect vacuum, etc. $[1,6]$. The result of these processes is a low current of charged particles. If these charged particles accelerate towards the insulator and impact the surface, they cause secondary electron emission. Then, if these initial secondary electrons restrike the insulator surface, a cascade of secondary electrons may evolve $[9,10]$.

Our approach differs from common practice in that we design an electromagnetic environment that causes charged particle trajectories to avoid the breakdown initiating impact on the surface of insulators and the development of a secondary electron cascade. In addition, we attempt to shape the electric fields along the insulator surface so that even if secondary electrons are formed by some unpredictable primary process, these will not be allowed to restrike the insulator at an upstream position and initiate a multipacting cascade. This approach leaves no rules of thumb in vacuum insulation but a theoretical road map to design which has to be implemented with care for each case studied. We have used this approach in our study of flat surface high-gradient insulators (HGI ) [11] where our theoretical approach was supported by experiment. HGI's have superior insulation properties [12] and are planned to be used as the accelerating tube of the planned DWA (dielectric wall accelerator) [13].

Our approach to vacuum-insulation design is not perfect because geometrical imperfections, surface charging, insulator surface irregularities, collisional gas ionization, the formation of plasma, etc. may complicate matters. However, we attempt to affect the initiation of breakdown before these complicated and difficult to predict processes develop. Thus, our methodology applies best to pulsedpower systems and more relevant for short pulses. If the voltage pulse is too long, preventing the initial steps of the breakdown may not be sufficient.

In the present paper we first show that for certain commonly applied techniques to improve voltage holdoff, the mechanism responsible for preventing vacuum surface breakdown follows our theory. Thus, in Sec. II A we discuss conical walled insulators while in II B we explain how stacked-ring insulators work [14]. Then, in II C, we model a case where stacked rings are used as a rule but can in fact be replaced by monolithic insulators (II D). Finally, in II E, we study sensitivities to mechanical tolerances. These studies allow us to present our approach by a set of practical examples.

We perform two types of calculations: charged particle ray tracing [15] in cases where time dependences are less important; particle in cell (PIC) calculations where these are required; or both if necessary. Without loss of generality, in all of our calculations we use dielectric materials with $\epsilon_{r} \sim 3$, which is close to the dielectric constant of plastic insulators most commonly used in vacuum pulsedpower technology. The reason that plastic insulators are more common in pulsed-power technology than, say, ceramics or glass, is because of the often large sizes required, the special shapes which need to be filled, and the rugged 
mechanical environment. In many cases, the insulator is required to separate the vacuum from large volumes of insulating oil with relative dielectric constant close to $\sim 3$ so the boundary between the liquid and solid insulator is electrically smooth.

\section{CASE STUDIES IN VACUUM INSULATION}

\section{A. Coned flat surface insulators}

Coning the shape of an insulator surface is known to improve voltage holdoff by a factor of up to $\sim 5$ as the cone angle increases to $\sim\left| \pm 45^{\circ}\right|$ (relative to the line perpendicular to the parallel cathode and anode surfaces) [16]. As the angle increases above $\sim\left| \pm 50^{\circ}\right|$, the breakdown threshold decreases.

In Fig. 1 calculated [17] equipotential curves are drawn for a typical situation with a coned surface insulator for a small and a large cone angle. When the insulator is perpendicular to the parallel cathode and anode surfaces, the electric field is parallel to the insulator surface, $E_{\|}$. For a coned surface, close to the surface of the insulator, in addition to the parallel component $E_{\|}$, the electric field has a component $E_{\perp}$ perpendicular to the surface. $E_{\perp}$ decreases as one departs from the insulator surface but as the cone angle increases, the effective range of the perpendicular component increases. In Figs. 1(a) and 1(b), we track trajectories of electrons initiated on the cathode wall close to the triple junction for cone angles $3.8^{\circ}$ and $45^{\circ}$, respectively. The small angle case [Fig. 1(a)] is similar to the perpendicular case (defined as $0^{\circ}$ ) where these electrons graze the surface of the insulator. If the surface is irregular, unsmooth, or contaminated, multipacting breakdown can certainly develop. By increasing the angle [Fig. 1(b)], $E_{\perp}$ increases and it deflects these electrons away from the surface so that they cannot restrike the surface and develop into a secondary electron breakdown cascade.

The electrons in Fig. 1(a) can produce secondary electrons while grazing the surface near the cathode end. In Figs. 1(c) and 1(d), we calculate the trajectories of secondary electrons emitted along the entire surface caused either by the impact of energetic electrons originating from processes such as whiskers exploding on the cathode surface, ionization of remnants of gas molecules close to the surface, or positive ion impact from whisker explosions, or gas ionization on or close to the anode. The electrons in 1(c) continue to graze the surface so that a breakdown secondary electron cascade is possible. As the cone angle increases [Fig. 1(d)], secondary electrons formed on the surface of the insulator are deflected away and cannot restrike the insulator surface.

In Fig. 2 we have drawn $E_{\|}$and $E_{\perp}$ along a line $100 \mu \mathrm{m}$ away from and parallel to the insulator surface in vacuum for the system in Fig. 1 for three values of the cone angle. As the angle increases, the magnitude of the parallel electric field component decreases, whereas the magnitude of the perpendicular component increases along most of the insulator surface. This is responsible for the secondary electron dynamics drawn in Fig. 1.

At higher angles, the region close to the anode becomes a "crowded corner" because the surface of the insulator is closer to the anode surface; both components of the electric field are stronger at the anode end, resulting in strong positive ion acceleration towards the now close insulator surface and electron acceleration away from it. In this crowded corner charged particles produced by nongeometric effects such as collisional ionization of trapped gas remnants and anode surface whisker explosions can more easily damage the closely lying insulator surface and initiate breakdown.

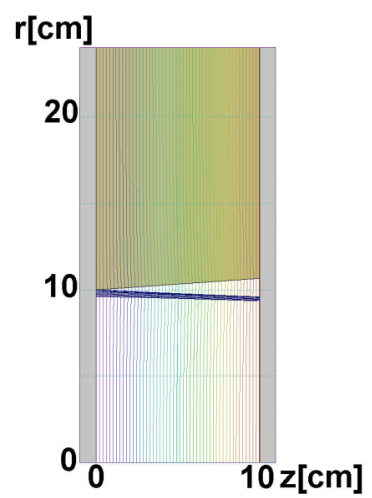

(a)

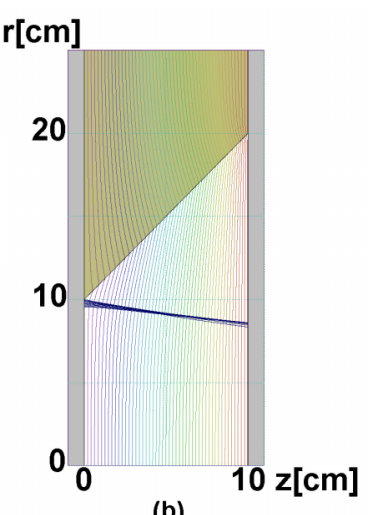

(b)

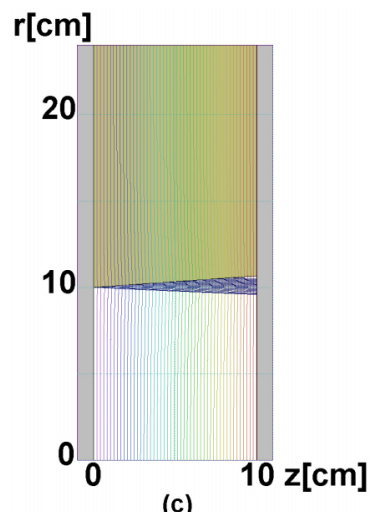

(c)

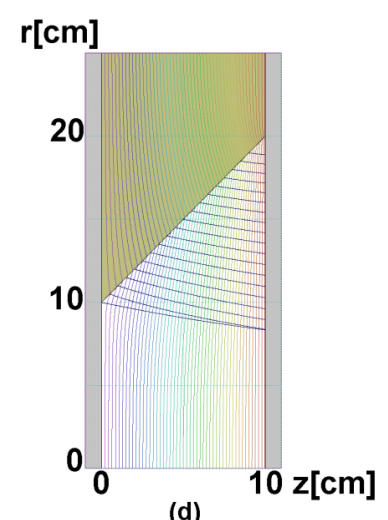

(d)

FIG. 1. (Color) Equipotential curves and electron trajectories (rays from left to right) in vacuum in a half cross section of a coned axially symmetric insulator ring (olive colored). The potential is applied between two parallel plates attached to the ends $z=0$ and $10 \mathrm{~cm}$ of the drawn device. Cone angles are $3.8^{\circ}$ [in (a) and (c)], $45^{\circ}$ [in (b) and (d)], $\left\langle E_{Z}\right\rangle=100 \mathrm{kV} / \mathrm{cm}$ and $\varepsilon_{r}=3$. In (a) and (b), ray traced trajectories of electrons emitted on the cathode surface near the triple junction are drawn. In (c) and (d), trajectories of electrons originating on the surface of the insulator are shown. 


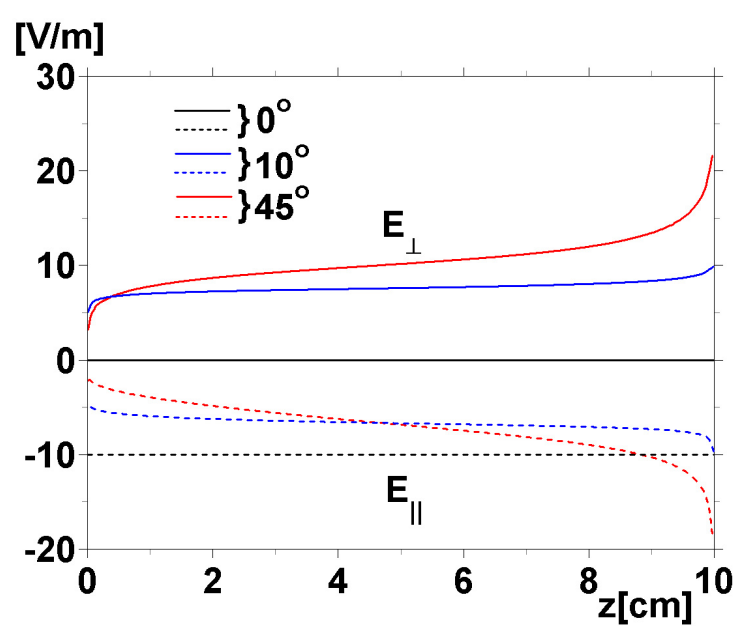

FIG. 2. (Color) $E_{\|}$(dashed) and $E_{\perp}$ (full) along a line $100 \mu \mathrm{m}$ away from the insulator surface in vacuum for the system in Fig. 1 with an anode-cathode separation of $10 \mathrm{~cm}, 1 \mathrm{~V}$ applied voltage, and $\varepsilon_{r}=3$ for three cone angles.

There has been considerable theoretical effort to calculate the electric field near triple junctions [18-20] and calculations of the type shown in Fig. 2 have been found to agree with theory [21]. Even though the fields near the anode triple junction for a cone angle of $45^{\circ}$ are much higher than for the perpendicular case $\left(0^{\circ}\right)$, the experimental breakdown field is $\sim 5$ times higher [16] which means that the dominant breakdown mechanism is that shown in Fig. 1. As the effect of "crowding" at the anode end becomes important the breakdown threshold field strength can be further affected by decreasing the fields at the anode end. Stygar et al. [2] have found that they could increase the voltage hold off of a $45^{\circ}$ coned insulator in their application by an additional factor of $\sim 1.7$ by adding a conducting "plug" at the anode end which decreases the electric fields at and near the anode triple junction. Decreasing the forces acting on surfaces, molecules and charged particles, due to electric fields causes particle dynamics in the "crowded" corner to be less energetic which decreases the probability to produce breakdown.

For negative angles, $E_{\perp}$ is negative and secondary electron emission is suppressed. As the value of the negative angle increases, the value of $E_{\perp}$ increases so that the strength of secondary electron emission suppression increases with the increasing value of the negative angle along most of the insulator surface. With a further increase in the value of the negative angle, the cathode surface becomes close to the insulator surface at the cathode end so that nongeometric processes may increase the breakdown probability initiated at the now crowded cathode end. The breakdown threshold fields for negative angles are lower than for positive angles because, though secondary electron emission is suppressed, the insulator surface attracts negatively charged particles and the surface may be damaged in a recurring test procedure.
We have used our theoretical methodology to explain the mechanism which decreases the probability for vacuum surface breakdown to occur for coned surface insulators. We have explained that by increasing the cone angle the probability for the development of a secondary electron breakdown cascade is considerably reduced because of the electric field shape close to the insulator surface. The reduction of the magnitude of the electric field is effective in the region of crowded corners. Experimental evidence $[1,16]$ shows that, at higher positive angles, processes near the anode triple junction become more important.

\section{B. The stacked-ring insulator}

Coning the insulator surface is difficult where large vacuum gaps need to be separated. Such a large insulator needs to be either cast or machined from bulk material. A large insulator mold for casting is usually very expensive. Machining a large coned surface insulator is difficult and large amounts of material are wasted. The stacked-ring insulator (stack) [5] solves these practical problems. In Fig. 3, a typical stack is drawn. A stack is made up of insulator rings separated by conducting metal rings. When a voltage is applied between the anode and the cathode (Fig. 3), each conducting ring sustains a floating constant potential according to the appropriate geometric division of the potential. Each insulator ring is coned individually to $+45^{\circ}$ so that the entire device remains cylindrical. This way the advantage of the cone angle with respect to vacuum breakdown is achieved without the disadvantages of coning a large monolithic insulator. The stack in pulsed high power systems ordinarily separates insulating oil (region above the stack in Fig. 3) from vacuum (Fig. 3region below the stack). Because of this, in each period of the stack a couple of O-rings are needed and insulator rods (not shown in Fig. 3) placed in the oil hold the contraption together. Thus, the stacked-ring insulator is a clever but complicated device.

Figure 3 shows that the mechanism responsible for the suppression of vacuum insulator breakdown in a stack follows our methodology. We are interested only in the vacuum region and in Fig. 3(a) we allow electrons representing whisker explosions on the cathode surface to be emitted along the cathode wall. The electric field shaping caused by the half rounded edged metal ring at the cathode end and the entire device does not allow electrons originating at the cathode end to reach the stack. In Fig. 3(b) we allow electrons to be emitted from all surfaces of the stack. These represent secondary electrons formed if any of these surfaces are hit by charged particles causing secondary emission. We see that electrons formed on the insulator rings behave similarly to those in Fig. 1(d) and no breakdown can develop along any one of the insulator rings. Electrons emitted from the rounded conductor edges are deflected away from the stack and no cascade develops. 


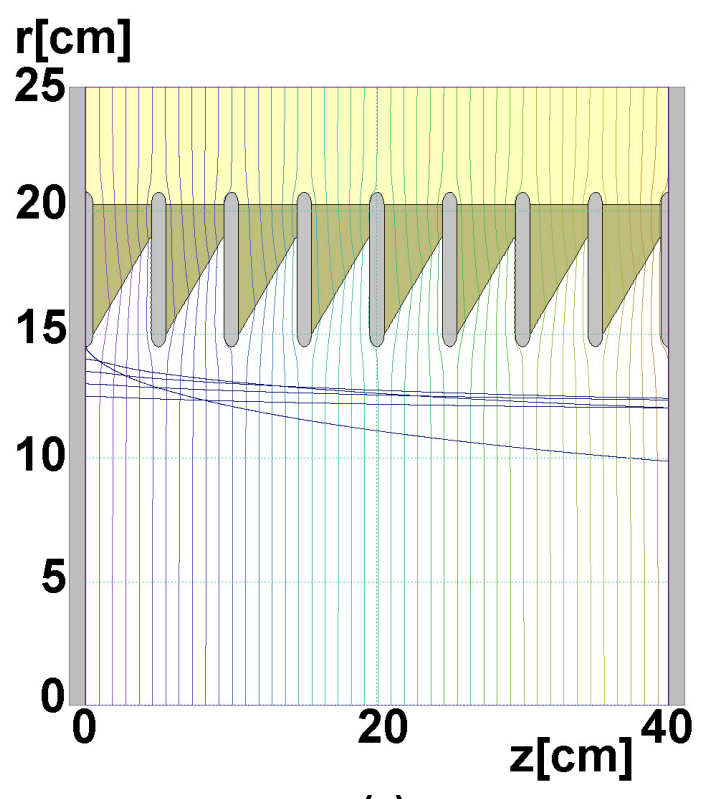

(a)

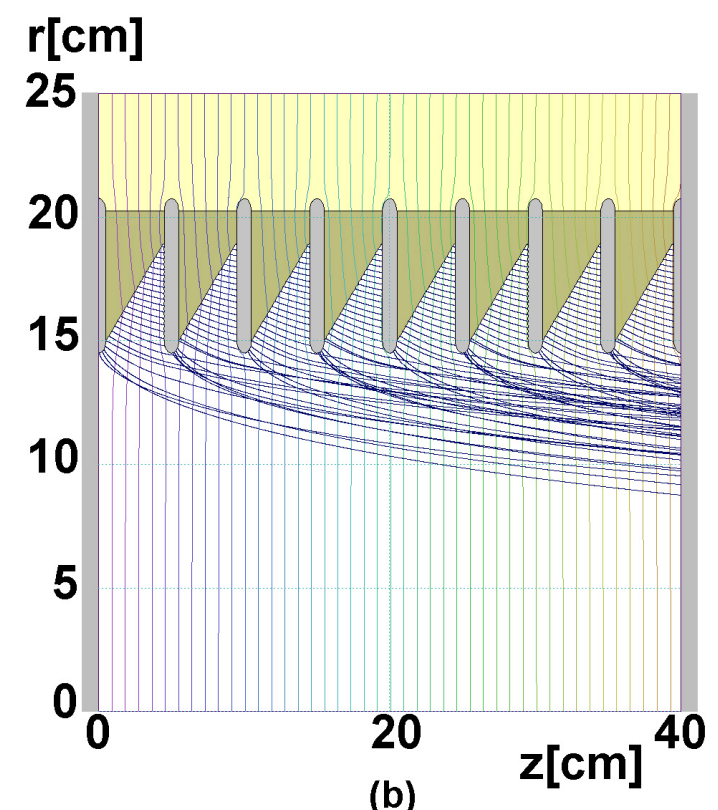

(b)

FIG. 3. (Color) Half cross section of an axially symmetric stacked-ring insulator between the parallel cathode (left gray end surface) and anode (right gray end surface). All gray surfaces are conductors, olive colored surfaces are insulators $\left(\varepsilon_{r}=3\right.$ ), and the yellow area is filled with insulating oil $\left(\varepsilon_{r}=2.7\right)$. Electrons (blue trajectories) flow from left to right. In (a) electrons are emitted on the cathode surface. In (b) the trajectories of all possible secondary electrons emitted along the surfaces of the stack are calculated and drawn. The lines almost parallel to the side plates are calculated equipotential curves for $1 \mathrm{MV}$ applied voltage.

None of these particles can initiate vacuum surface breakdown showing that the stack is an effective device.

\section{The stacked-ring insulator at the front of a pulsed-power machine}

The front of a typical pulsed-power machine is one situation where stacks are commonly used [22]. In Fig. 4 we can see a typical front end of a pulsed-power machine attached to a transmission line which supplies power but is represented in our ray-tracing calculations by a constant voltage between the anode and the cathode. In all our calculations we apply a voltage of $2-2.5 \mathrm{MV}$.

In Fig. 4 we separate the insulating oil from the vacuum region with a monolithic insulator tube. We allow low current electrons to evolve from two regions. Electrons originating on the cathode wall close to the triple junction graze the surface and are ultimately attracted to the insulator. Electrons originating on the conducting central stalk are accelerated towards the insulator surface. These processes may cause damage to the insulator and vacuum surface breakdown may become unpredictable when the surface is damaged.

Experience guides designers of such systems to replace the insulator in Fig. 4 with a stack [22] (Fig. 5). In Fig. 5 we can see that the cathode end electrons accelerate towards upstream conducting rings. Their impact may cause secondary electron emission and damage only on the edge of the conducting rings. Depending on the design of the rings, breakdown may stop on a certain conducting ring or at most continues but only between the edges of the metal rings without damage to the insulator. The difference between the application seen in Fig. 5 and that seen in Fig. 3 is due to the central stalk which distorts the electric field between the parallel anode-cathode structure so that electrons are deflected towards the stack. Electrons originating on the central stalk can impact the stack or graze the insulator rings resulting in secondary electron emission (not shown). These are accelerated towards adjacent up-

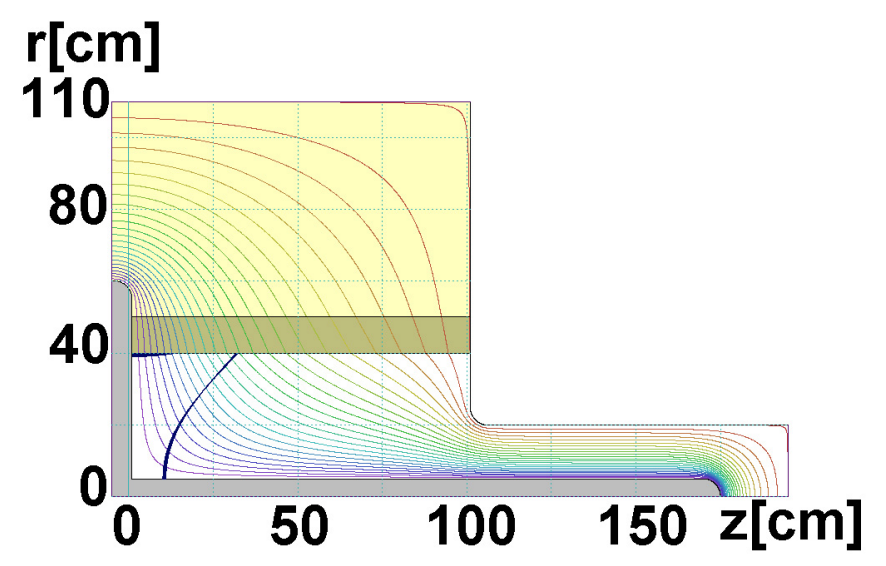

FIG. 4. (Color) Equipotential curves at the front end of a typical pulsed-power machine (half cross section of the axially symmetric structure is drawn). Low current electrons are emitted at two "weak" points: at the cathode triple junction and along the central stalk. Parts are colored as in Fig. 3. 


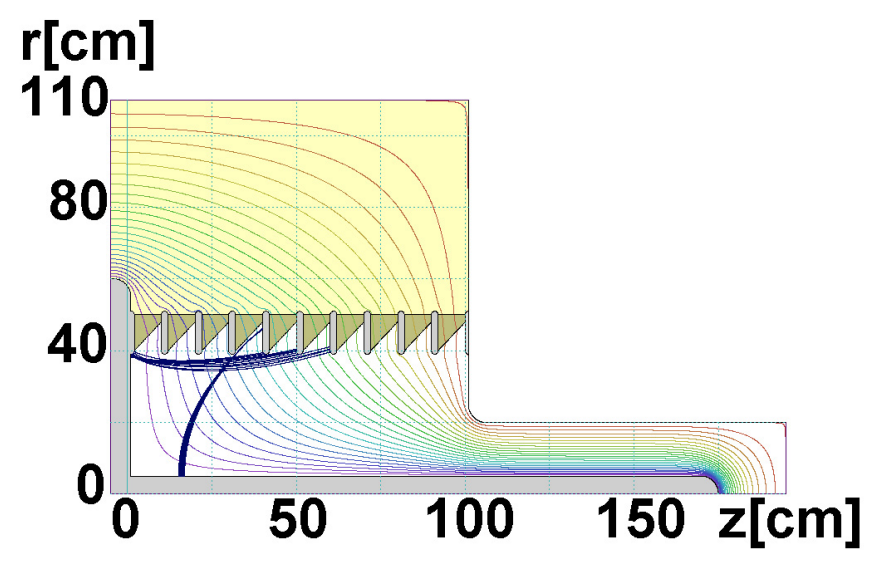

FIG. 5. (Color) Same as Fig. 4 but the insulator is replaced by a stack.

stream metal rings without causing breakdown because of the local effect of the insulator cone angle. In contrast to the situation of Fig. 3, with a central stalk, the stack can be damaged by electrons originating from discharges on the central stalk but this is probably not critical in practice because damage is at most localized to a specific ring.

So far, we may summarize that replacing the monolithic insulator with a stack at the front of a pulsed-power machine is favorable in the sense that vacuum surface breakdown may be avoided. In Sec. II D we show that a stack it is not necessarily the optimal choice.

In pulsed-power systems of the type described above, a voltage pulse is applied and space charge limited emission is possible between the cathode and the anode. Then a current develops along the central stalk which in turn produces an azimuthal magnetic field, $B_{\theta}$, which can affect the motion of the primary breakdown electrons shown in Fig. 5. The TRAK code [23] allows the calculation of $B_{\theta}$ while calculating the current shown in Fig. 6.

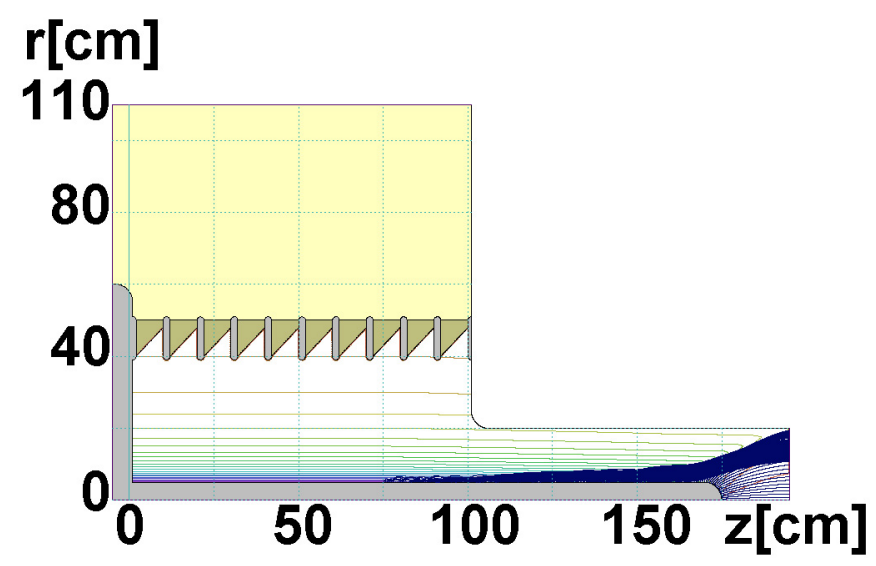

FIG. 6. (Color) TRAK $[14,15]$ ray-tracing calculation of the space charge limited emission from the central stalk of the device of Fig. 5. Contours of the beam generated $B_{\theta}$ are also drawn. 2.4 MV is applied and the total current obtained is $44 \mathrm{kA}$.
We propagate the breakdown initiating electrons shown in Fig. 5 in the presence of the beam generated magnetic fields produced in the calculations shown in Fig. 6. Beam generated electric fields are also calculated and accounted for. The results of this calculation are seen in Fig. 7 where $B_{\theta}$ deflects the electrons away from the stack.

In Fig. 8(b) the electrons shown in Fig. 4 are propagated in the presence of the beam generated magnetic and electric fields calculated with a monolithic insulator [Fig. 8(a)]. Grazing the insulator surface over a few $\mathrm{cm}$ is retained even in the presence of $B_{\theta}$. This can be damaging to the insulator and possibly initiate surface breakdown.

The above calculations represent two different situations. First, at early times before $B_{\theta}$ grows, breakdown along a monolithic insulator may develop (Figs. 4 and 5). Once current flows between the cathode and the anode, $B_{\theta}$ deflects those electrons which are possible surface breakdown initiators. A stack is in general advantageous over a monolithic insulator.

To complete this picture we performed PIC calculations for the above systems. We apply a $10 \mathrm{nsec}$ rise time voltage pulse at the open boundary of the above discussed systems. The pulse is allowed to develop until steady state is achieved with respect to the voltages and the currents within the system. We allow electrons to be emitted from all metallic surfaces in vacuum above a threshold electric field of $230 \mathrm{kV} / \mathrm{cm}$ (red particles in all following PIC results). Electrons are also emitted at a negligible threshold with low initial currents (yellow particles) from the same regions as those chosen in Figs. 4 and 5. In Fig. 9(a) the voltage pulses measured during the PIC calculation of the system shown in Fig. 9(b) are drawn. The time delay represents the wave propagation while steady state is achieved after $\sim 35$ nsec. $V_{3}$ is noisy because the measurement is performed in the presence of the PIC charged particles beam.

In Figs. 10(a)-10(c) snapshots of the electron phase space at $t=7.003,9.664$, and $45.007 \mathrm{nsec}$, respectively,

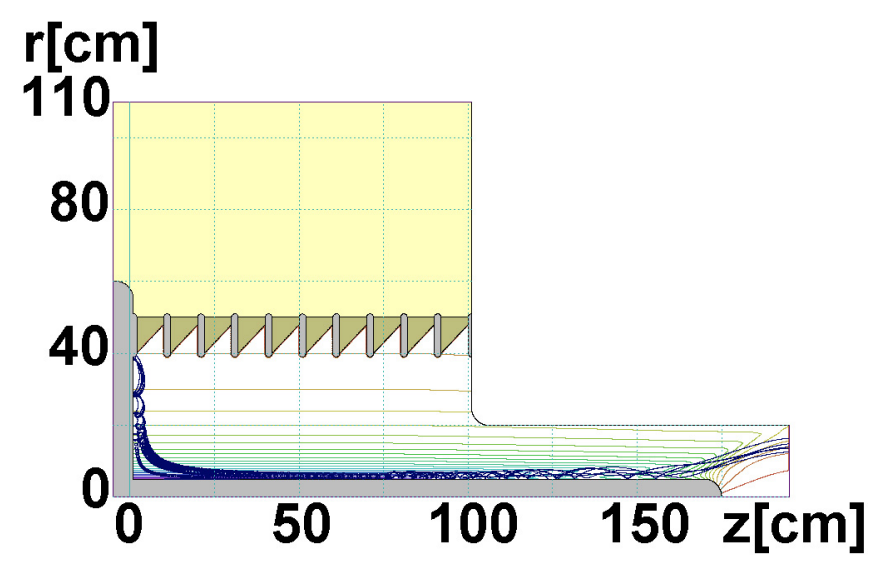

FIG. 7. (Color) The trajectories of the electrons shown in Fig. 5 in the presence of the beam generated magnetic and electric fields as calculated in Fig. 6. 


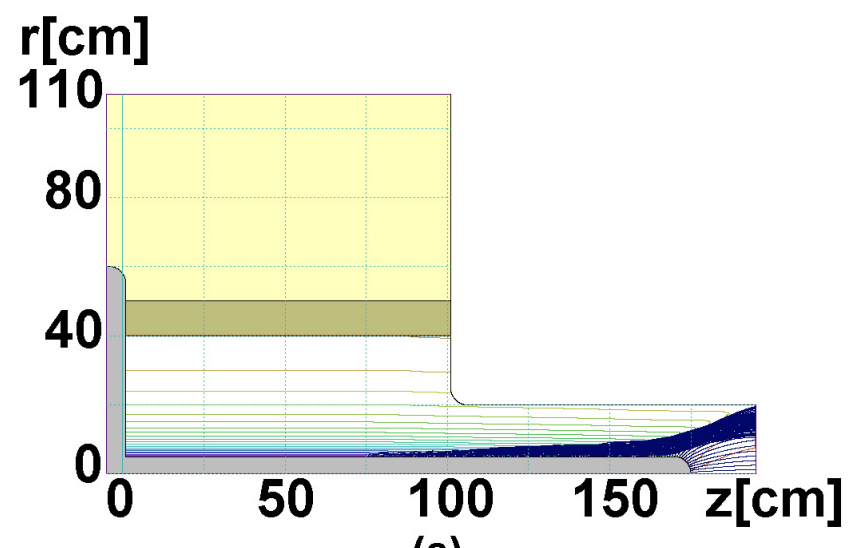

(a)

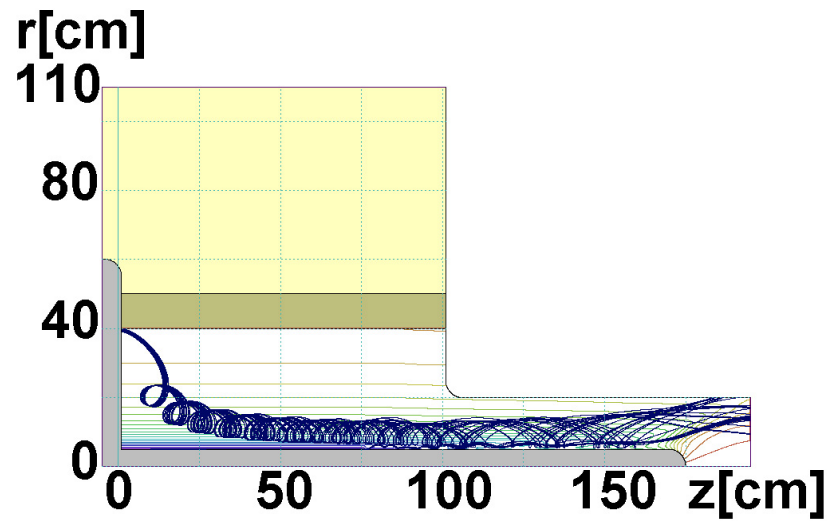

(b)

FIG. 8. (Color) Part (a) is the same as Fig. 6 for a monolithic insulator and part (b) the same as Fig. 7 for the electrons shown in Fig. 4.

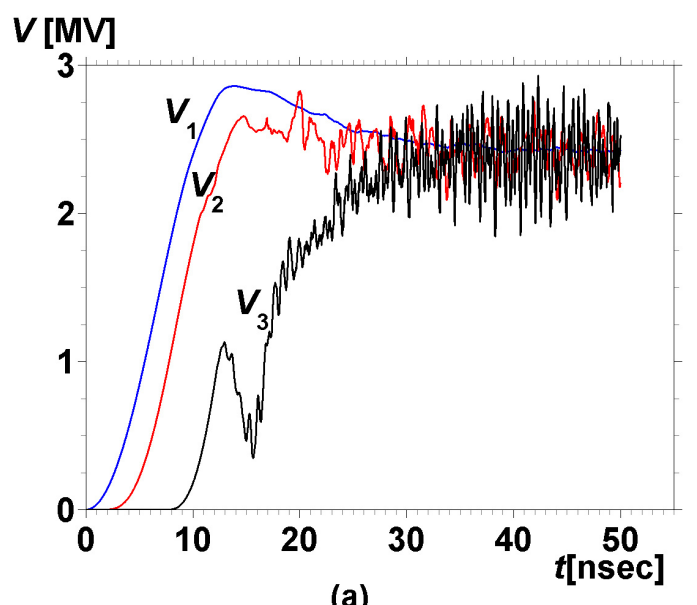

(a)

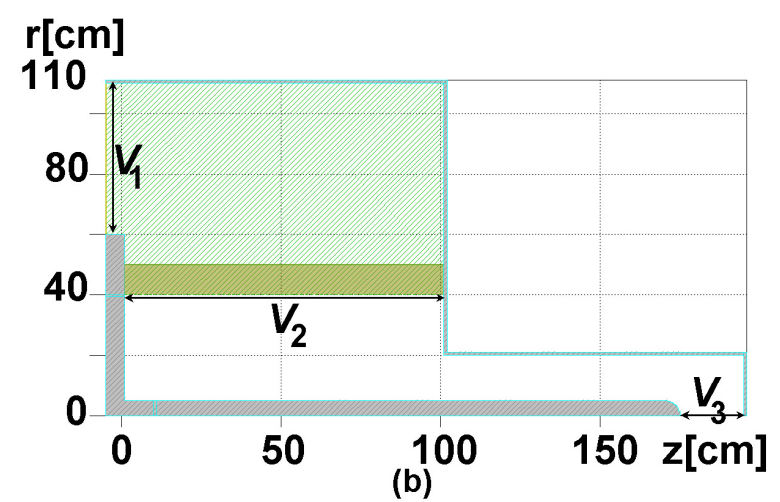

(b)

FIG. 9. (Color) The voltage vs time (a) as measured at various locations during the PIC calculation performed on the system shown in (b). The geometry of the PIC calculations in $9(\mathrm{~b})$ is the same as that in Fig. 4.

are shown. Figures 10(a) and 10(b) are at times within the rise time of $V_{2}$ and display similar behavior to that seen in Fig. 4. In 10(b) a current starts to flow between the cathode and the anode which produces enough $B_{\theta}$ to deflect electrons emitted from the stalk. Finally, 10(c) is clearly comparable to Fig. 8. In Fig. 10(d) we have drawn a snapshot of the charge density distribution at the same time as the phase space in Fig. 10(c). The charge density close to the insulator is much smaller than close to the stalk. Thus, at steady state the electron flow close to the insulator is small compared to the rest of the device but during the initial stages, vacuum surface breakdown along the monolithic insulator can occur, justifying the conclusions drawn from the electrostatic calculations of Fig. 4. If breakdown develops at early times the late time calculations of Fig. 10 become irrelevant.

The series of PIC calculations shown in Fig. 11 are the same as Fig. 10 but with a stack replacing the monolithic insulator. The behavior in Figs. 11(a) and 11(b) is very similar to that seen in Fig. 5. Though 11(c) is slightly different than Fig. 7, there is no difference in the nature of the particle motion. The quality of the ray tracing TRAK calculations becomes even more pronounced when the values of the calculated currents are compared to the PIC currents at the steady state voltage. In the above calculations the steady state voltage was $\sim 2.4 \mathrm{MV}$ for both the monolithic and stack insulators with steady state total PIC currents of 43.4 and $43.5 \mathrm{kA}$, respectively. The corresponding TRAK calculated currents were 42.3 and $44.0 \mathrm{kA}$. Taking account of the fact that the two types of calculations are extremely different in nature, the comparison both in terms of the calculated currents and the nature of the charged particle dynamics is remarkable.

We can conclude that our calculations which represent our methodology support the common practice of using a stack rather than a monolithic insulator at the front of a pulsed-power machine even when a central stalk is present. 


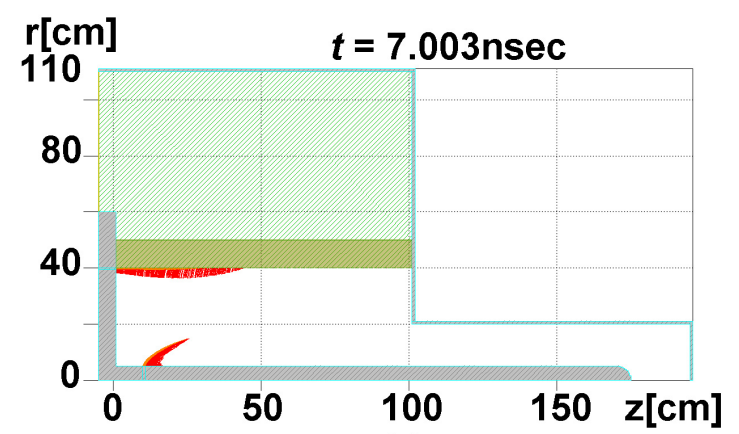

(a)

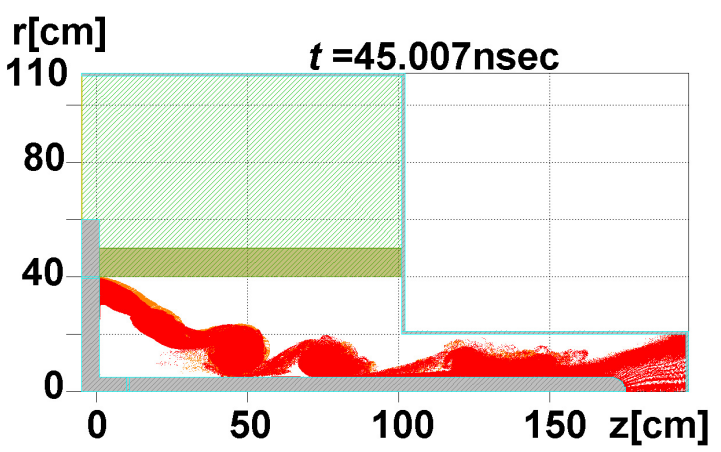

(c)

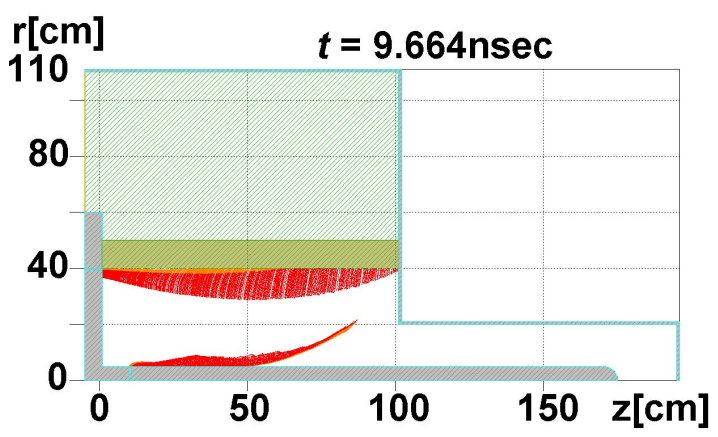

(b)

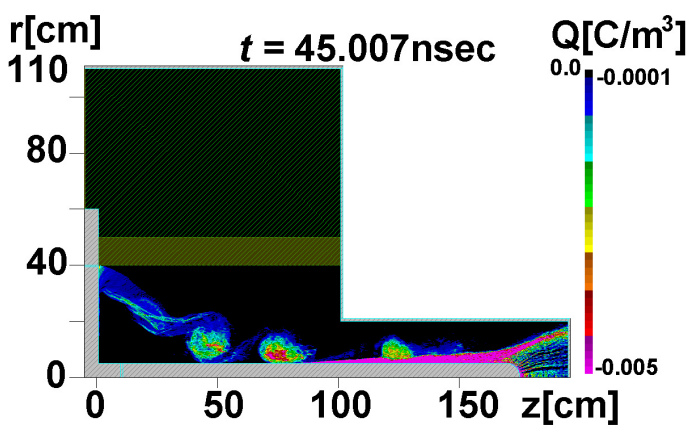

(d)

FIG. 10. (Color) PIC calculated phase space of all electrons at $t=7.003$ (a), 9.664 (b), 45.007 (c) nsec for the system of Fig. 9(b). In (d) charge density contours are drawn for the same instant as the phase space in (c).

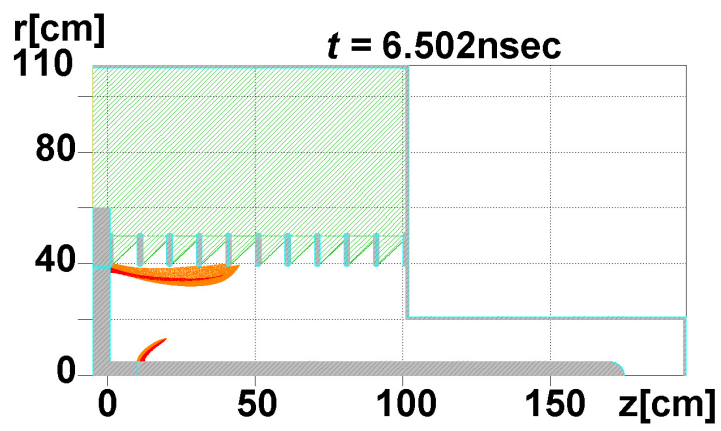

(a)

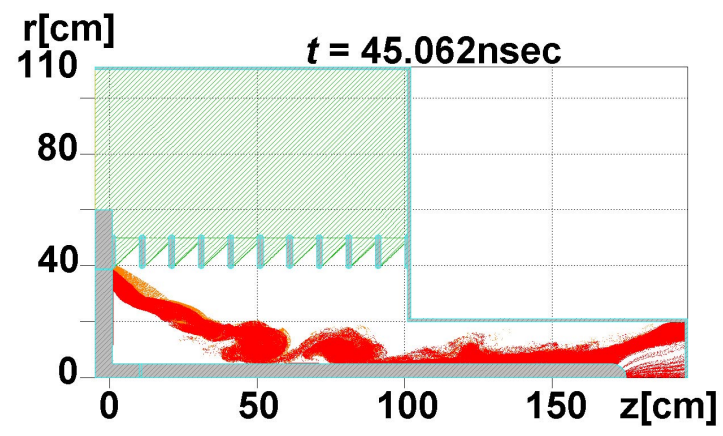

(c)

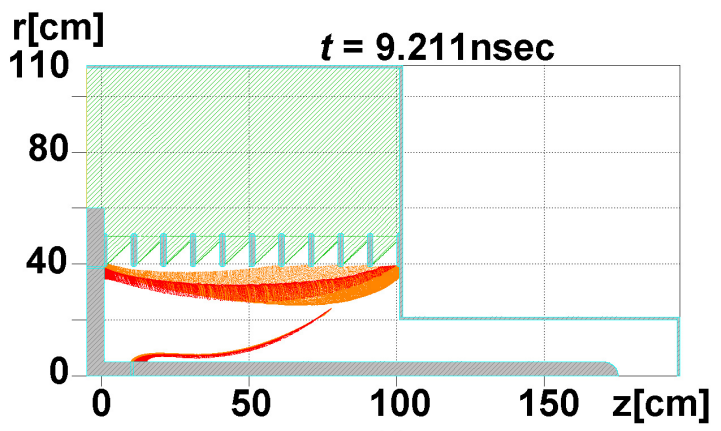

(b)

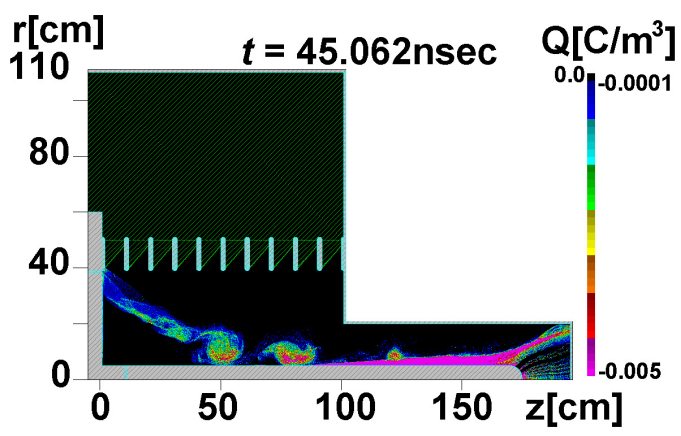

(d)

FIG. 11. (Color) Same as Fig. 10 with a stack replacing the monolithic insulator for $t=6.502$ (a), 9.211 (b), 45.602 (c) nsec, and charge density contours in (d) at 45.062 nsec. 


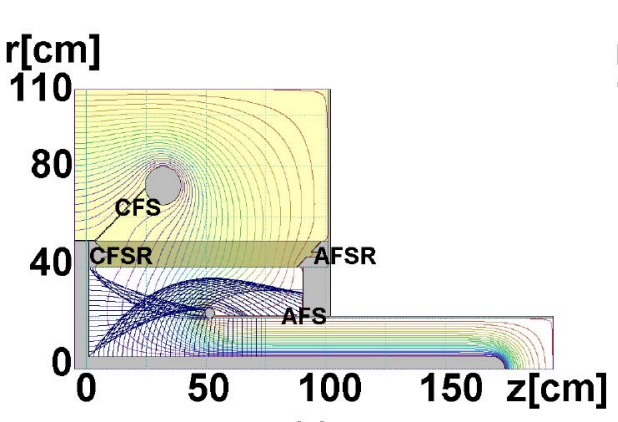

(a)

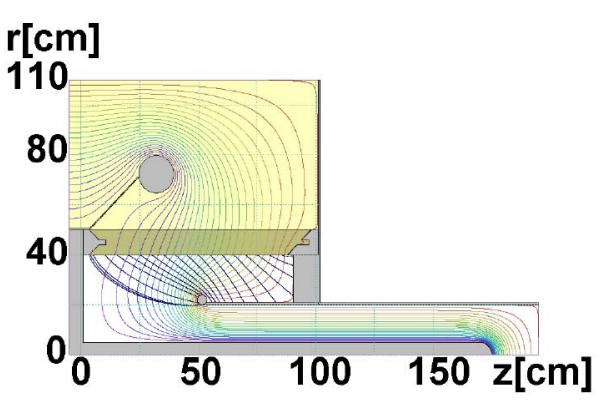

(b)

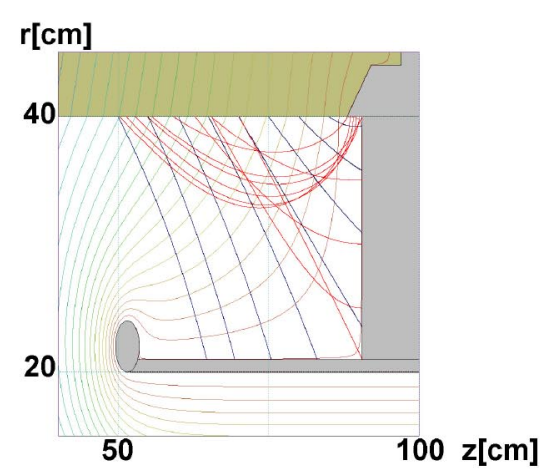

(c)

FIG. 12. (Color) Ray-tracing calculations at the front of a pulsed-power machine with a central stalk. The knob in the cathode fieldshaping ring (CFSR) and the notch in the anode field-shaping ring (AFSR) are simple attempts to add mechanical strength but have a negligible EM effect. In (a) electrons (blue trajectories) originating on the cathode surfaces, in (b) secondaries emitted along the monolithic insulator, whereas in (c) positive ions (red trajectories) emitted at the enlarged anode end are traced. Contours of the voltage distribution are also drawn.

\section{A monolithic insulator and appropriate field shaping at the front of a pulsed-power machine}

In this section we propose a design [14] in which for the pulsed-power machine of Sec. IIC a monolithic insulator can be used, provided the electric field is shaped along the insulator in such a way that vacuum flashover is avoided. This is achieved in Fig. 12 by adding various parts to shape the electric field along the insulator in such a way as to prevent surface grazing of electrons emitted near the cathode triple junction, prevent electrons emitted from the stalk impact the insulator (see Fig. 4), and prevent the formation of a crowded corner near the anode triple junction, while at the same time keeping the design simpler than a stack. In Fig. 12(a) our design consisting of a cathode field shaper (CFS), an anode field shaper (AFS), a cathode fieldshaping ring (CFSR), and an anode field-shaping ring (AFSR) is drawn. Figure 12 represents the results of ray tracing of electrons emitted from various surfaces just as we did in previous sections.

Figure 12(a) shows that the effect of the cathode triple junction can be eliminated by adding a conducting ring collinear to the insulator at the cathode end. This is similar to the half-period conducting ring we used at the cathode end in HGI's [11]. Electrons originating on the cathode field-shaping ring are accelerated away towards the anode field shaper without causing harm. In fact, none of the electrons emitted on the cathode surfaces have a chance to reach the insulator. These are collected either on the anode field shaper or the anode itself. If by some chance charged particles do impact the insulator and secondaries are emitted on the insulator then [Fig. 12(b)], these are accelerated away from it without being able to restrike the insulator and cause breakdown. The reason for this is clearly seen in Fig. 13 where we have drawn the components of the electric field close to the insulator surface. $E_{\perp}$ is positive along most of the insulator length just as if it were coned to a positive angle (Fig. 2). There are two distinct differences between Fig. 13 and Fig. 2. First, the effect of the cathode field-shaping ring is seen as $E_{\perp}$ is now positive and nonzero beyond the cathode triple junction. This causes all electrons around this point to be accelerated away from the surface. Second, $E_{\perp}$ is negative at the anode end due to the inclusion of the anode field-shaping ring. This suppresses electron emission and at the same time avoids positive ions from grazing the surface of the insulator at the anode end. In Fig. 12(c) positive ions originating on the anode field-shaping ring and on the anode surface are traced. These do not graze the surface though eventually impact the insulator surface. Consequently, secondary electrons (blue lines) emitted from the impact points on the insulator will simply be accelerated away from the surface. Thus, no considerable secondary cascade can develop.

The value of the electric field at the anode end in Fig. 13 is quite strong; nonetheless, the direction of the fields and

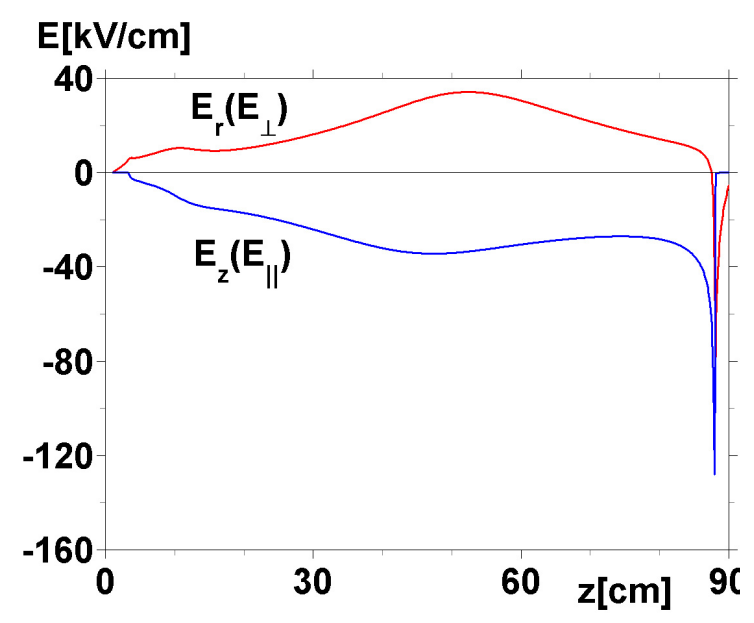

FIG. 13. (Color) Values of the components of the electric field with 2.2 MV applied voltage $10 \mu \mathrm{m}$ below the monolithic insulator in Fig. 12 vs $z$. 


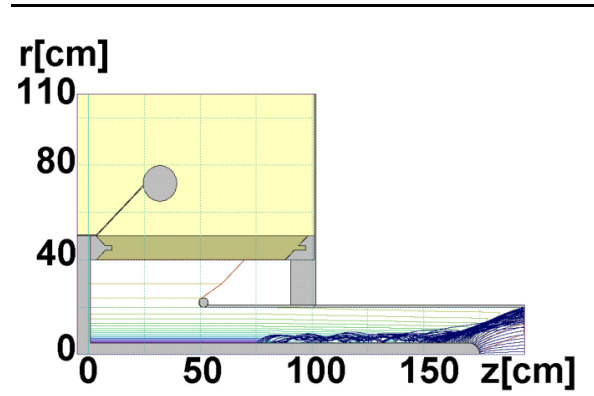

(a)

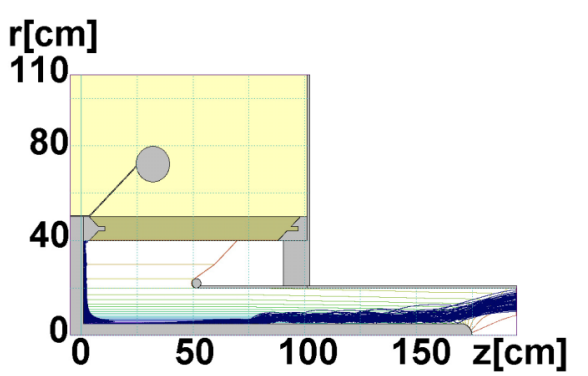

(b)

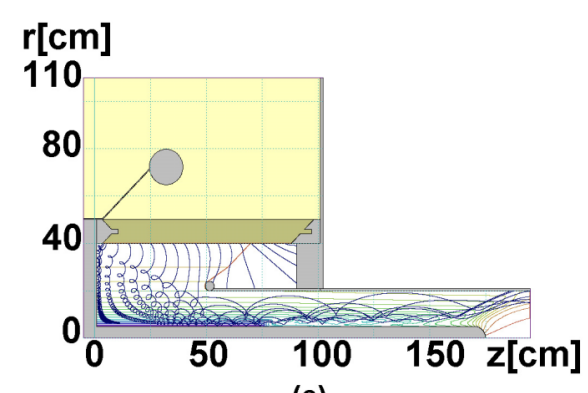

(c)

FIG. 14. (Color) Space charge limited emission of an electron beam current from the cathode stalk in (a) generates magnetic and electric fields. In (b) the low current electrons originating on the cathode field-shaping ring and the central stalk are propagated in the presence of the fields generated in (a). In (c) secondaries of the type shown in 12(b) are traced in the presence of these fields.

the dynamics of the charged particles prevent the development of breakdown. The anode end of the present insulator design does not constitute a crowded corner as is the case with a coned insulator. Cathode and anode end fieldshaping rings have been successfully utilized with HGI's [11].

In Fig. 14 we test the effect of the beam generated magnetic field when a current develops between the cathode and anode [Fig. 14(a)] on the trajectories of the electrons of Fig. 12 for an applied voltage of 2.2 MV. The results of Fig. 14 show that, if breakdown is prevented at early times when the stalk current is not yet developed as predicted by our design in Fig. 12, then, with the development of the beam generated magnetic field, breakdown has little probability to occur. Positive ion motion is not affected by the magnetic fields.

To support this picture, we performed a complete time dependent PIC calculation for this case too. In Fig. 15 we display the time dependence of the voltage as measured at the same positions as those shown in Fig. 9(b). In Fig. 16 we present snapshots of the electron phase space at various instants of time. As in Figs. 10 and 11, we allow low threshold emission from the cathode field-shaping ring, the cathode triple junction region, and along the central stalk at $z=0.1 m$ (yellow particles in Fig. 16).

Figure 16 shows that the design prevents electrons from approaching the insulator surface even at the early times before the self-magnetic field has developed just as in Fig. 12(a). As the magnetic field increases, the low current electrons are deflected even further away. The similarities between Fig. 14 and Fig. 16 are remarkable. Moreover, the current of the beam calculated by ray tracing in Fig. 14 was $42 \mathrm{kA}$ for the applied 2.2 MV whereas the PIC calculations shown in Figs. 15 and 16 steady at $\sim 35 \mathrm{nsec}, V=$ 2.2 MV, and give a beam current of $39.5 \mathrm{kA}$.

In this section we have used our theoretical methodology to design a monolithic insulator at the front of a pulsedpower machine on which vacuum surface breakdown is prevented by appropriate electric field shaping. The electric field is shaped in such a way that primary charged particle impact on the insulator surface is prevented and secondary electrons cannot restrike the insulator. We have found that ray-tracing steady state methods are sufficient as a calculating tool for such a design. Calculations need to be performed first without high current beam generated forces. These represent the early time development stages. To prove that a design is appropriate, both these and calculations including beam generated forces need to show that electrons are deflected away from insulator surfaces and that if secondary electrons are produced then these have little probability to restrike the surface.

The calculations performed here suggest that, as long as charged particle impact on the insulator surface is prevented by field shaping, it is not necessary to increase the length of the insulator with increasing voltage to the extent required by statistical considerations [1,24]. With stacks, because of the proximity of the conducting ring tips to the central stalk, as the voltage needs to be increased, the stack diameter needs to be increased [24]. With field shaping, electron impact on a monolithic insulator's surface is prevented and its diameter may not be required to be increased considerably if the voltage needs to be increased. Experiments are needed to test our design and verify these state-

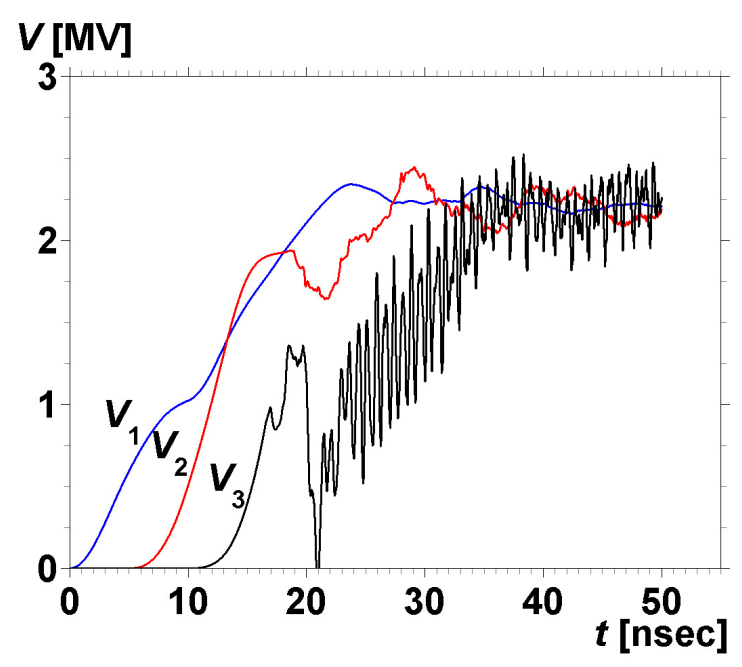

FIG. 15. (Color) Same as Fig. 9(a), for the geometry of Fig. 14. 


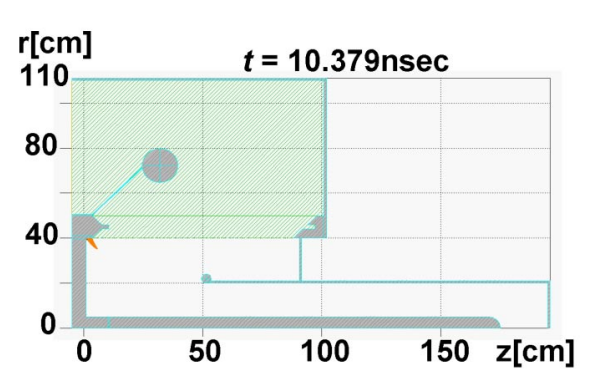

(a)

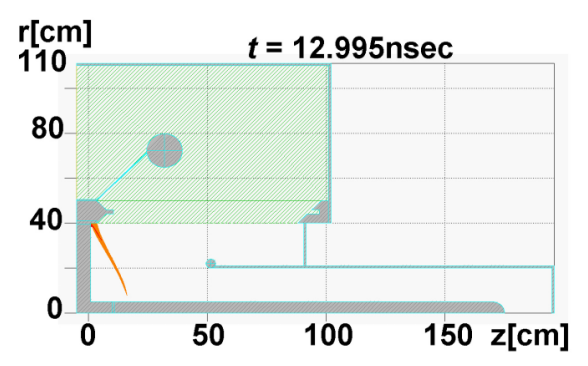

(b)

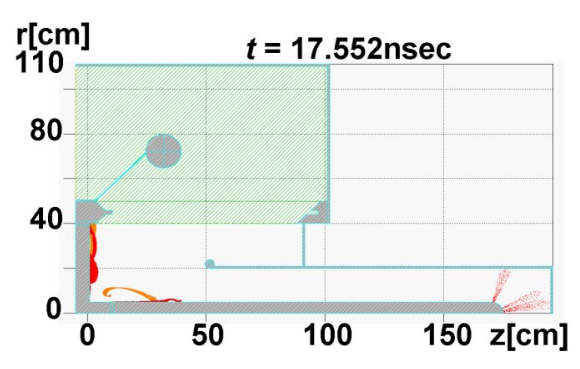

(c)

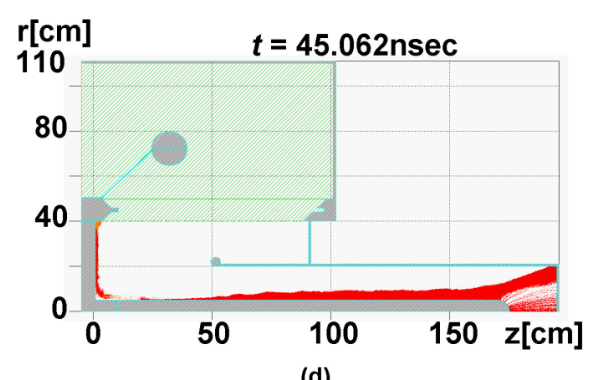

(d)

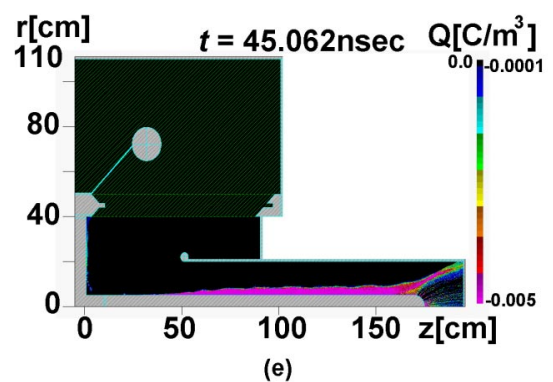

FIG. 16. (Color) PIC calculated phase space of all electrons at $t=10.379$ (a), 12.995 (b), 17.552 (c), and 45.062 (d) nsec for the system of Fig. 14. In (e) the charge density contours are drawn at the same time instant as in (d).

ments which could be of importance because it means that the voltage sustained by such pulsed-power machines is not necessarily limited by stack size.

\section{E. The effect of mechanical tolerances on the design of vacuum insulation}

In the previous section we have shown that the cathode and anode field-shaping rings are important as they change the nature of the end triple junctions and shape the nearby fields so that electron and ion motion is directed in such a way that vacuum surface breakdown is prevented. In practice, an ordinary triple junction of any type can never be manufactured to be on a perfect line. We think that these imperfections are often the source for the initiation of breakdown. In the present section we shall study the effect of some of these mechanical inaccuracies on triple junctions of the type designed along the insulator of the previous section. We shall show that our methodology is helpful with respect to this aspect too.

In Fig. 17 we study the motion of secondary electrons near a flat surface cylindrical insulator tube with a cathode field-shaping ring attached to it. This ring has been used in HGI's [11] and in a modified shape in the previous section. Figure 17 represents a very accurate ray-tracing calculation of part of a structure close to the triple junction. The full structure studied is a $10 \mathrm{~cm}$ long insulator ring with a $1 \mathrm{~cm}$ long field-shaping ring attached to its cathode end though the behavior scales and the dimensions are not that important [11]. As we have seen before, in Fig. 17(a) if charged particles impact the surface, secondary electrons are deflected away from the surfaces surrounding the triple junc- tion. In Fig. 17(b) we allow the conducting ring to protrude in the vacuum $0.1 \mathrm{~mm}$, while in 17 (c) the conductor is recessed below the insulator surface. These represent practical mechanical inaccuracies which may occur within tolerances defined for the collinearity of the surfaces. In Fig. 17(b) some electrons are seen to graze the surface of the insulator close to the now different type of cathode triple junction formed due to the surface mismatch. In Fig. 17(c) electrons originating on the cathode fieldshaping ring may impact the insulator corner and cause damage or negative charging. This may be perhaps better than the grazing in Fig. 17(b) but highly unpredictable as to how breakdown may be affected. Surface imperfections of the type seen in Fig. 17 occur on flat surface HGI's because of the way the surface consisting of two materials of different strengths is cut by a water jet [25]. Figure 17 shows that a mismatch can change the behavior of the secondary electron dynamics and consequently the performance of the insulator.

In Sec. II D we have designed the cathode field-shaping ring with a $45^{\circ}$ local cone angle (Fig. 12). In Fig. 18 we show the reason for this choice and how this choice is affected by mechanical restrictions. In Fig. 18(a) we see that a cone angle of $45^{\circ}$ affects secondary electrons emitted from the insulator surface only slightly and electrons are removed from the surface in a similar manner as in Fig. 17(a). When the conductor protrudes into the vacuum as in Fig. 18(b), electrons no longer graze the surface of the insulator [Fig. 17(b)], whereas when it is recessed as in Fig. 18(c) the situation is worse than in Fig. 17(c) near the now crowded triple junction formed by the surface irregu- 


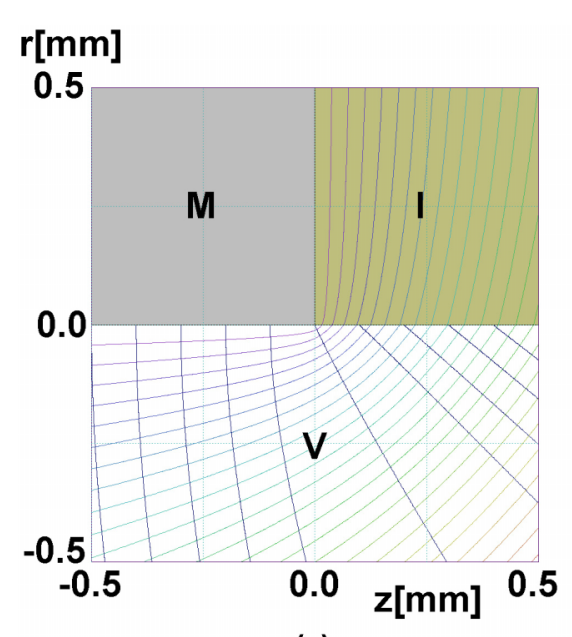

(a)

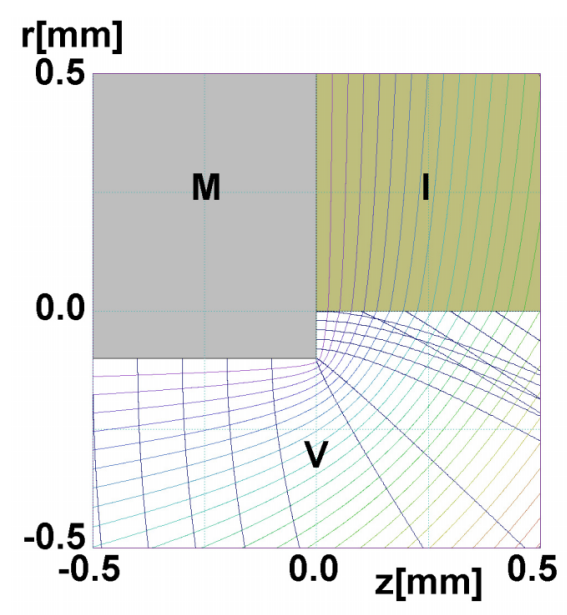

(b)

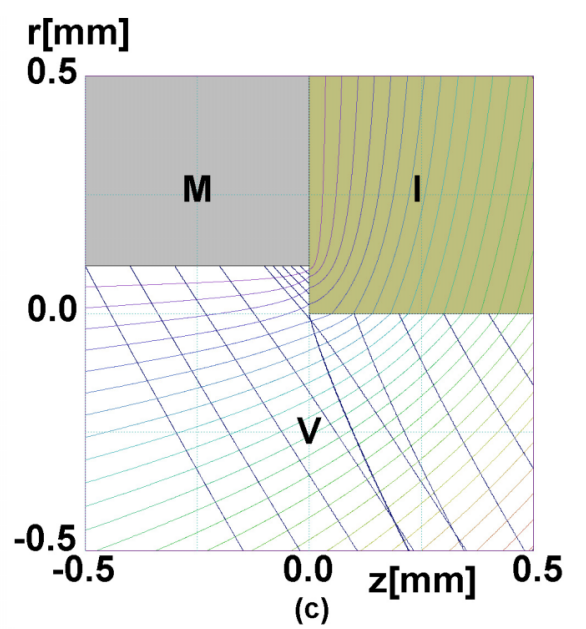

FIG. 17. (Color) Ray traced secondary electrons emitted from surfaces near the cathode triple junction for (a) collinear surfaces, (b) with the conducting ring protruding $0.1 \mathrm{~mm}$ into the vacuum above the insulator surface, and (c) with the conducting ring recessed $0.1 \mathrm{~mm}$ beneath the insulator surface. $M, I$, and $V$ designate regions of conducting material, insulator, and vacuum, respectively. $\varepsilon_{r}=2.7$. The colors represent contours of fixed potential and the applied voltage was $2000 \mathrm{kV}$ over a device length of $10 \mathrm{~cm}$, that is $\left\langle E_{z}\right\rangle=200 \mathrm{kV} / \mathrm{cm}$. Electron trajectories move away from the surfaces into the vacuum.

larity. For the cathode field-shaping ring of Fig. 17, we cannot allow surface mismatch, which is impractical, whereas, for the one in Fig. 18 we can define a practical tolerance such that the metallic ring should be collinear or protruding.

The electric fields in the vicinity of the triple junction in Fig. 17(a) are higher than those in 18(a). Nevertheless, beside the mechanical advantage described above, the two cases are comparable in terms of preventing breakdown.

In Fig. 19 we allow the anode field-shaping ring's surface to mismatch the insulator surface. When the surfaces are collinear the behavior of the charged particle dynamics is seen in Fig. 12(c). The choice of this part's shape is governed by the requirement to remove ions away from the surface. In Fig. 19 we show both ion trajectories (red) originating on the anode field-shaping ring and secondary electrons (blue) emitted on the insulator surface. In Fig. 19(a) a crowded corner is formed when the conducting ring's surface protrudes into the vacuum, whereas if this ring is recessed beneath the insulator, ions do not impact the insulator near the triple junction [Fig. 19(b)]. For the anode end, the anode field-shaping ring should not protrude into the vacuum beneath the insulator surface, in contrast to the cathode end.

In the above discussion, we have compared the effect on vacuum surface breakdown of various surface misalignment situations near some triple junctions occurring due to mechanical tolerances. Following the analysis we were able to narrow the required tolerances. We have, though,

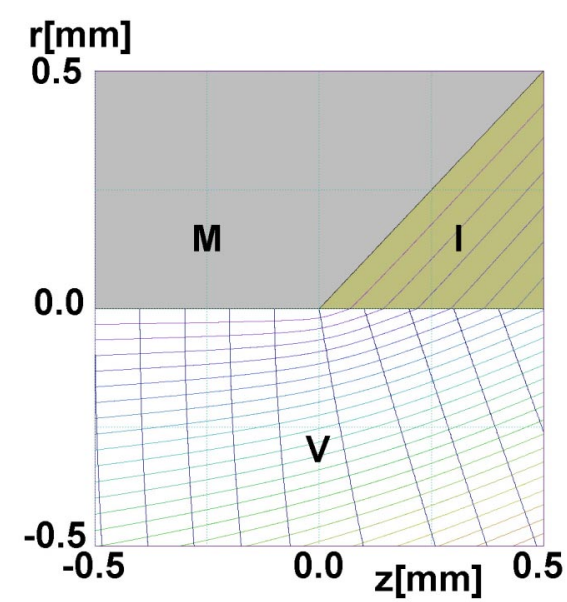

(a)

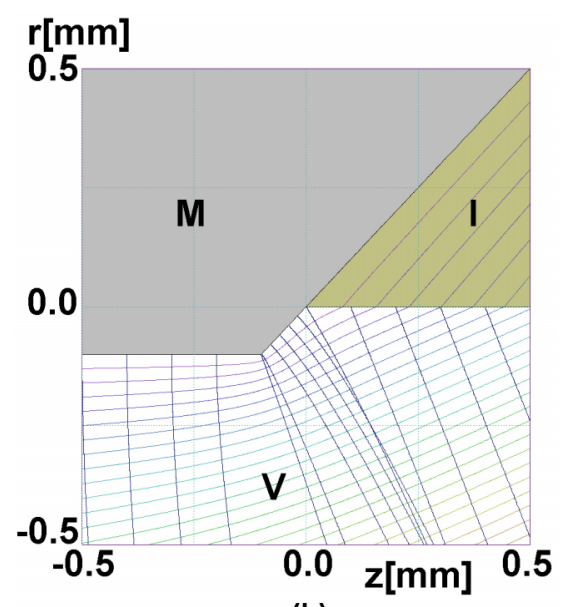

(b)

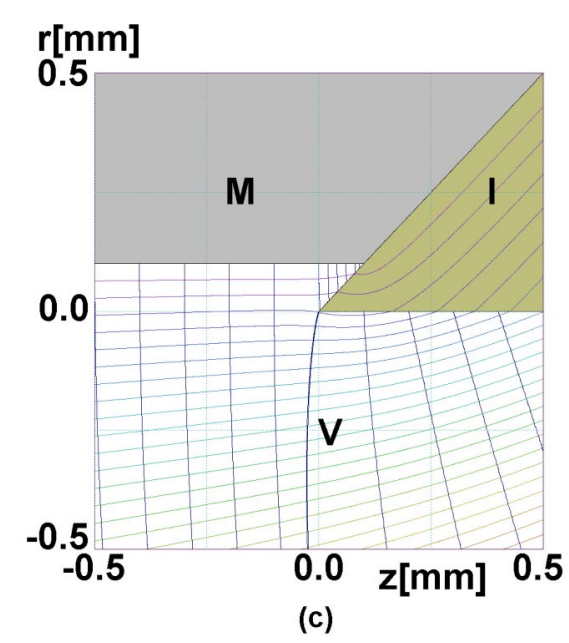

(c)

FIG. 18. (Color) Same as Fig. 17 but with the conductor-insulator interface coned to $45^{\circ}$. 


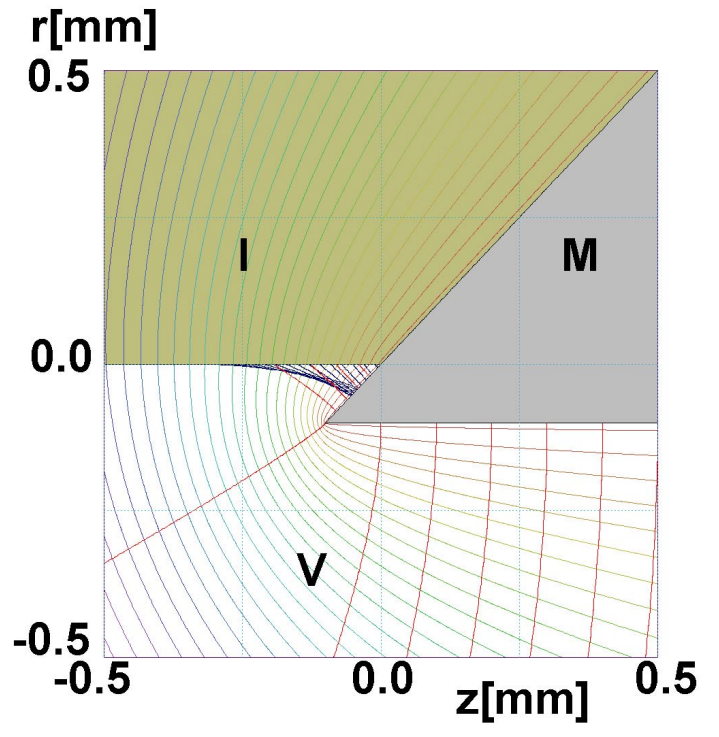

(a)

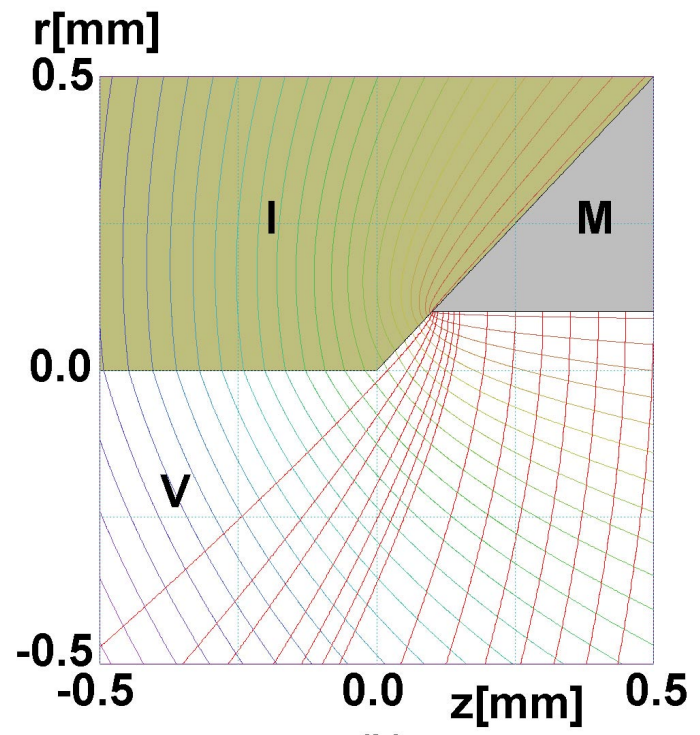

(b)

FIG. 19. (Color) Same as Figs. 17 and 18 but for the anode field-shaping ring. Positive ion trajectories are drawn as red curves in the vacuum.

assumed that the triple junction lies on a perfect line which is impossible in practice. In Fig. 20 we take the analysis a step further. We compare an ordinary cathode triple junction, that is, an insulator ring attached directly to a perpendicular conducting cathode wall to the flat surface cathode triple junction of Fig. 17(a). In Fig. 20(a) we assume that the insulator ends in a rounded edge of radius $0.1 \mathrm{~mm}$ resulting in a void between the cathode and the insulator surfaces. In Fig. 20(b) we assume that the edges of both the conducting ring and the insulator are rounded forming a similar void. In both figures we emit electrons from the surface of the conductor in and near the resulting void. Figure 20(a) explains the reason behind the statement that an ordinary cathode triple junction is the prime reason for the initiation of vacuum surface breakdown. The electric fields for this situation attract electrons to the surface of the

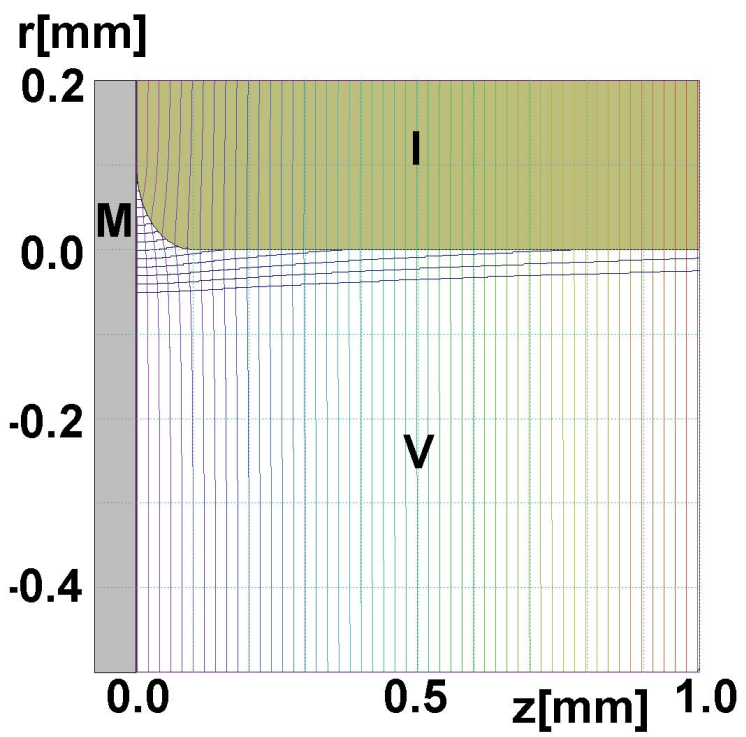

(a)

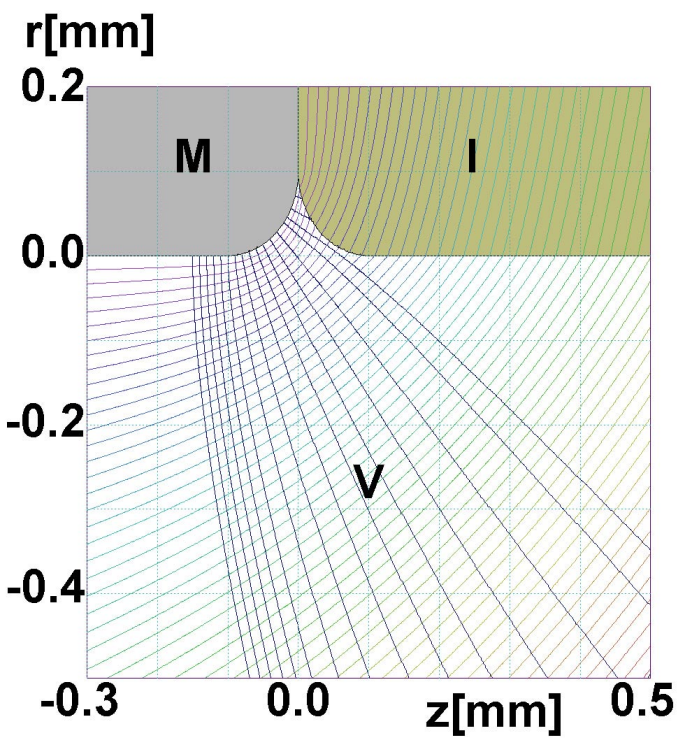

(b)

FIG. 20. (Color) Ray traced electrons emitted on the cathode surface near an imperfect triple junction. In (a) an ordinary cathode triple junction is studied whereas in (b) an imperfect flat cathode triple junction of the perfect type seen in Fig. 17(a). The imperfections are introduced by rounding specific corners to a radius of $0.1 \mathrm{~mm}$. All other parameters are the same as in Fig. 17. 
insulator. Some electrons graze the surface more than if the motion were only parallel to the surface and may initiate breakdown. Other electrons are attracted to the insulator wall at the edge of the void. Though secondary emission is suppressed, the impact may cause surface damage. The situation is much better in Fig. 20(b) where most electrons are accelerated away from the surface. Changing the cone angle of the conductor-insulator interface as in Fig. 17 can improve this picture further.

\section{SUMMARY}

In this paper we have presented a theoretical methodology for design of vacuum insulation in high-voltage pulsed-power systems. We suggest that, by shaping the electromagnetic fields in such a way that charged particles produced initially are deflected from impacting the surface, vacuum surface breakdown can be prevented. Charged particles can be deflected from insulator surfaces even if electric fields are strong at various locations on the insulator surface. As long as distances are kept long enough so that no breakdown occurs in a system due to lack of dielectric strength, the mechanism proposed here does not depend on insulator length. Increasing the breakdown length is a statistical requirement which takes care of the unpredictable part of vacuum surface breakdown. Our methodology decreases the unpredictability because we introduce control of some of the processes involved. This affects the extent to which the length of the insulator is required to be proportional to the applied voltage.

We have shown that quite complex modeling is required, from ray-tracing techniques to PIC calculations, in order to follow charged particle trajectories. We think that this theoretical methodology is more appropriate in vacuum-insulation design and that calculating and decreasing electric fields on or near dielectric surfaces is not sufficient. So far our methodology has been successfully demonstrated in experiments on HGI's - more experiments will be needed. Nonetheless, we do not claim to provide any rules of thumb in vacuum insulation but propose that the methodology presented here should be applied with care for each case studied and that some of the mechanical tolerances need to be addressed as these may affect the mechanism.

[1] H. C. Miller, IEEE Trans. Electr. Insul. 24, 765 (1989).

[2] W. A. Stygar, J. A. Lott, T. C. Wagoner, V. Anaya, H. C. Harjes, H. C. Ives, Z. R. Wallace, G. R. Mowrer, R. W. Shoup, J. P. Corley, R. A. Anderson, G. E. Vogtlin, M.E. Savage, J. M. Elizondo, B. S. Stoltzfus, D. M. Andercyk, D. L. Fehl, T. F. Jaramillo, D. L. Johnson, D. H. McDaniel, D. A. Muirhead, J.M. Radman, J.J. Ramirez, L.E. Ramirez, R. B. Spielman, K. W. Struve, D. E. Walsh, E. D. Walsh, and M.D. Walsh, Phys. Rev. ST Accel. Beams 8, 050401 (2005).
[3] B. A. Prichard, Jr., J. Barraza, M. Kang, K. Nielsen, F. Bieniosek, K. Chow, W. Fawley, E. Henestroza, L. Reginato, W. Waldron, R. Briggs, T. Hughes, and T. Genoni, Particle Accelerator Conference, Knoxville, Tennessee, 2005, edited by C. Horak, p. 169, IEEE Catalog No. 05CH37623C.

[4] R. W. Shoup, F. Long, T. H. Martin, W. A. Stygar, H. Ives, R. B. Spielman, K. W. Struve, M. Mostrom, P. Corcoran, and I. Smith, Proceedings of BEAMS'96, Prague, 1996, edited by K. Jungwirth and J. Ullschmied (Czech Academy of Science, Prague, 1996), p. 989.

[5] J.C. Martin on Pulsed Power, edited by T.H. Martin, A. H. Guenther, and M. Kristiansen (Plenum, New York, 1996).

[6] S. Humphries, Principles of Charged Particle Acceleration (Wiley, New York, 1989), Chap. 9.5.

[7] J.P. VanDevender, D. H. McDaniel, E. L. Neau, R.E. Mattis, and K.D. Bergeron, J. Appl. Phys. 53, 4441 (1982).

[8] J.D. Cross and T.S. Sundarshan, IEEE Trans. Electr. Insul. 9, 146 (1974).

[9] P. A. Chatterton, in Electrical Breakdown in Gases, edited by J. M. Meek and J.D. Craggs (Wiley, New York, 1978).

[10] A. S. Pillai and R. Hackam, J. Appl. Phys. 53, 2983 (1982).

[11] J. G. Leopold, U. Dai, Y. Finkelstein, E. Weissman, and S. Humphries, IEEE Trans. Dielectr. Electr. Insul. 12, 530 (2005).

[12] S. E. Sampayan, P. A. Vitello, M. L. Krogh, and J. M. Elizondo, IEEE Trans. Dielectr. Electr. Insul. 7, 334 (2000).

[13] G. J. Caporaso, S. Sampayan, Y.-J. Chen, J. Harris, S. Hawkins, C. Holmes, S. Nelson, A. Paul, B. Poole, M. Rhodes, D. Sanders, J. Sullivan, L. Wang, J. Watson, M. Krogh, W. Nunnally, and K. Selenes, Proceedings BEAMS'2006, Oxford, UK (to be published).

[14] J. G. Leopold, I. Be'eri, E. C. Levi, C. Leibovitz, and I. Navon, Proceedings BEAMS'2006, Oxford, UK (to be published).

[15] S. Humphries, in Computational Accelerator Physics, edited by R. Ryne (AIP, New York, 1994), p. 597. (All ray tracing calculations in the present paper use the FIELD PRECISION TRICOMP group of codes developed by the S. Humphries. See www.fieldp.com.)

[16] O. Milton, IEEE Trans. Electr. Insul. 7, 9 (1972).

[17] J. G. Leopold, U. Dai, S. Zamir, and E. Wissman, Proceedings of the IEEE Pulsed Power Conference, Monterey, CA, 2005, edited by J.E. Maenchen and E. Schamiloglu, p. 509, IEEE Catalog No. 05CH37688C.

[18] L. Schachter, Appl. Phys. Lett. 72, 421 (1998).

[19] M.S. Chung, S.C. Hong, P.H. Cutler, and N.M. Miskovsky, J. Vac. Sci. Technol. B 22, 1240 (2004).

[20] M. S. Chung, S. C. Hong, P. H. Cutler, N. M. Miskovsky, B. L. Weiss, and A. Mayer, J. Vac. Sci. Technol. B 24, 909 (2006).

[21] L. K. Tully, D. A. Goertz, T. L. Houck, and J. B. Javedani, Report UCRL-TR-227505, 2006, pp. 1-5.

[22] R. J. Allen, G. Cooperstein, F. C. Young, J. W. Schumer, D. D. Hinschelwood, D. Mosher, D. Holmberg, and S. E. Mitchell, Proceedings of the IEEE Pulsed Power Conference, Dallas, TX, 2003, edited by Michael 
Giesselmann and Andreas Neuber, p. 883, IEEE Catalog No. 05CH37688C.

[23] S. Humphries and T. Orzechowski, Phys. Rev. ST Accel. Beams 9, 020401 (2006).

[24] I.D. Smith, Phys. Rev. ST Accel. Beams 7, 064801 (2004).
[25] R. A. Schill, Jr., W. Culbreth, R. Venkat, J. M. Elizondo, A. J. Dragt, and M. Krogh, Proceedings of the IEEE Pulsed Power Conference, Las Vegas, NV, 2001, edited by R. Reinovsky and M. Newton, p. 1798, IEEE Catalog No. $05 \mathrm{CH} 37688 \mathrm{C}$. 